\title{
Physical Activity and Exercise for Optimal Disease Prevention: Clinical Evidence \\ Editorial
}

Gabriel J. Sanders ${ }^{1}$

${ }^{1}$ Northern Kentucky University, Highland Heights KY, US A

Corresponding author: Gabriel J. Sanders, sandersg1@nku.edu

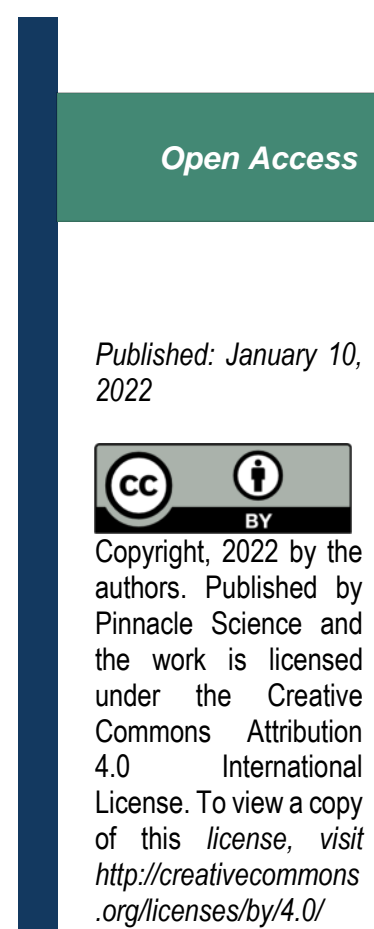

Research Directs in Health Sciences: 2022, Volume 2 (Issue 1): 1

ISSN: 2768-492X

Key words: preventative treatment, strategies, multiple factors

\section{Editorial}

Previous physical activity guidelines from health organizations provide general physical activity and exercise intensity and duration recommendations ${ }^{1}$. These guidelines have experienced very little change over the last two decades, despite significant changes in technology, more specifically wearable technology ${ }^{2}$. The guidelines typical refer to exercise intensity as low, moderate and vigorous intensity based on a metabolic equivalent scale (MET) or a subjective scale. With wearable technology being accessible, affordable, reliable, and accurate, more attention should be given address recommendations that are multifaceted and specific. Most wearable technology can easily track sleep, steps, calories, hear rate, and exercise time within certain heart rate training zones. Research has shown that monitoring exercise and physical activity with wearable technology can improve health outcomes ${ }^{3}$.

Future guidelines should aim to include specific information that can be easily tracked with wearable technology. Prior to specific guidelines be published, additionally clinically relevant data is needed to assess how specific step counts, caloric expenditures, and time engaged in heart rate ranges can positively benefit health outcomes such as heart disease and obesity. While much research has been conducted on the use of wearable technology ${ }^{2}$, a more narrow focus is needed for researchers to assess patient adherence and outcomes when exercise is prescribed based on heart rate and time engaged in various heart rate training zones ${ }^{2,4}$.

\section{Conclusion}

While epidemiological data and secondary data analysis regarding physical activity and exercise interventions are valuable, randomized control trials (RCT) with control arms consisting of specific exercise guidelines tracked by wearable technology with a goal of achieving multiple activity components, such as heart rate training zones, daily steps, and exercise time, would be of value to the literature. Combined data from multiple different RCTs would provide clinical evidence regarding the effectiveness of prescribing specific physical activity and exercise prescriptions that can be tracked on wearable technology. Utilizing this method, a participant or patient knows, in real time, if they are achieving the intensity and duration goals of the exercise prescription. It is time to move the needle on research in physical activity and exercise as a use of preventative medicine.

\section{References}

1. Wright DC, Swan PD. Optimal exercise intensity for individuals with impaired glucose tolerance. Diabetes Spectrum. 2001;14(2):93-97. 
2. Loncar-Turukalo T, Zdravevski E, Machado da Silva J, Chouvarda I, Trajkovik V. Literature on Wearable Technology for Connected Health: Scoping Review of Research Trends, Advances, and Barriers. J Med Internet Res. 2019;21(9):e14017.

3. El-Amrawy F, Nounou MI. Are Currently Available Wearable Devices for Activity Tracking and Heart Rate Monitoring Accurate, Precise, and Medically Beneficial? Healthc Inform Res. 2015;21(4):315-320.

4. Sanders GJ, Boos B, Rhodes J, Kollock RO, Peacock CA. Competition-Based Heart Rate, Training Load, and Time Played Above 85\% Peak Heart Rate in NCAA Division I Women's Basketball. J Strength Cond Res. 2018. 